\title{
Non-linear interaction of the kinetic Alfvén waves and the filamentation process in the solar wind plasma
}

\author{
R. P. Sharma and M. Malik
}

\author{
Center for Energy Studies, Indian Institute of Technology, Delhi 110016, India \\ e-mail: rpsharma@ces.iitd.ernet.in
}

Received 19 December 2005 / Accepted 16 May 2005

ABSTRACT

\begin{abstract}
We have considered two parallel propagating kinetic Alfvén waves (KAW) propagating in a plasma with $\beta$ in the limit $\left(m_{\mathrm{e}} / m_{\mathrm{i}} \ll \beta \ll 1\right)$. The spatial amplification and ultimately filamentation of a perturbation present on one of the KAW has been studied, especially in the presence of second KAW. Nonlinear evolution of the perturbation, present on the main KAW, into the filamentary structures and its dependence on various parameters of the second KAW has been investigated in detail for solar wind parameters. These filamentary structures can act as a source for the particle acceleration by wave particle interaction because the KAW are mixed modes and Landau damping is possible. The relevance of this work to the solar wind turbulence spectrum and the energization of heliosphere particles by the solar wind is discussed.
\end{abstract}

Key words. plasmas - Sun: solar wind

\section{Introduction}

Alfvén waves are ubiquitous in space plasmas. Alfvén waves are present in a number of astrophysical systems, including the region of space ahead of supernova shocks. They have been detected in the solar wind (Coleman 1966; Unti \& Neugebauer 1968; Belcher \& Davis 1971), and in the vicinity of bow shock waves at the Earth and other magnetized planets (Fairfield 1969; Hoppe \& Russell 1983). Alfvén waves are difficult to dissipate, hence believed to be good candidates for energy and momentum transfer. Alfvén waves can energize plasma if there exist a means, that can transfer the wave energy from longer spatial scales to shorter spatial scales (as classical dissipation is $\delta l^{-2}$, here $\delta l$ is the spatial scale length, Tsiklauri et al. 2005). One such means for transferring the energy from the large scale to the small scale is the transverse collapse mechanism (e.g. filamentation).

The solar wind is formed by the gas-dynamic expansion of the solar corona. In situ observations have revealed that the solar wind exists in two distinct states: the fast stream $\left(>700 \mathrm{~km} \mathrm{~s}^{-1}\right)$ and the slow $\left(\approx 400 \mathrm{~km} \mathrm{~s}^{-1}\right)$ stream. The low speed streams originate in the open fields above active regions of the solar corona. The high speed streams originate over coronal holes The predominance of nearly incompressible Alfvén waves in the solar wind stream has also been confirmed by Daily (1973), Parker (1980a,b) and Bavassano et al. (1982). Hollweg et al. (1982) analyzed data from the Helios 2 satellite and found Alfvén waves making a significant contribution to the high speed streams. A detailed discussion of Alfvén wave domination in the solar wind is given by Denskat \& Burlaga (1977). The Alfvén waves observed in situ in the solar wind provide a clue that dissipation of Alfvén waves in the solar corona could provide the heat input and wave pressure gradient (wave ponderomotive force) necessary to heat and accelerate the high speed solar wind (Parker 1991). Alfvén waves can be dissipated in the solar corona by magnetic reconnection, current cascade, MHD turbulence, Alfvén resonance, resonance absorption or phase mixing. A number of authors have investigated the heating and acceleration of the solar wind.

Ulysses and Helios observations have shown that in the high speed streams, the minor (heavy) ions flow faster than the protons by about the local Alfvén speed along the magnetic field and that they are hotter than the protons roughly in proportion to their masses. Remote observations by UVCS on SOHO indicate that in the vicinity of $3 R_{\mathrm{s}}$, the $\mathrm{O}^{5+}$ ions have a perpendicular temperature $T_{\perp} \sim 2 \times 10^{8} \mathrm{~K}$, while the protons have $T_{\perp} \sim 3 \times 10^{6} \mathrm{~K}$. Also the bulk flow speeds of $\mathrm{O}^{5+}$ are faster than the proton speeds where $r \geq 2 R_{\mathrm{s}}$. The heavy ions are strongly anisotropic in temperature. (See Hollweg \& Isenberg 2002, and references therein for a thorough review.) A number of authors have investigated the heating of the solar wind ions due to waveparticle interactions (e.g. Hollweg \& Turner 1978; Dusenbery \& Hollweg 1981; Marsch et al. 1982; Isenberg 1984; Leer et al. 1992; Hu et al. 1997; Czechowski et al. 1998; Cranmer et al. 1999).

A number of mechanisms for the acceleration of the solar wind have been investigated and are also able to explain many observations, but a mechanism that can explain all the observations is still lacking. The filamentation process (hot spot formation) may provide a clue to the dissipation problem, as it is a fast way to transport energy at small scales.

The Kinetic Alfvén wave (KAW) is the shear Alfvén wave modified by short wavelength effects, and it can be produced when an obliquely propagating shear Alfvén wave is affected by the electron temperature and inertia such that a non-zero parallel electric field arises within the wave itself due to the local charge separation (Lysak \& Lotko 1996; Wu 2003). The parallel component of the perturbed electric field can play an important role in accelerating and heating plasma particles. KAW has been discussed extensively in the fields of laboratory, space, and astrophysical plasmas since the pioneering theoretical work 
of Hasegawa \& Chen (1975). The importance of KAWs in explaining the physics of small scale auroral arcs has been widely accepted. Indeed, localized waveforms with scales of the order of $\sim V_{\mathrm{Ae}} / \Omega_{\mathrm{e}}$ or $\rho_{\mathrm{s}}\left(\Omega_{\mathrm{oe}}\right.$ is the electron gyrofrequency, and $V_{\mathrm{Ae}}$ and $\rho_{\mathrm{s}}$ are the Alfvén speed and ion Larmor radius, respectively. $\rho_{\mathrm{s}}=c_{\mathrm{s}} / \Omega_{\mathrm{p}}$, with $c_{\mathrm{s}}$ and $\Omega_{\mathrm{p}}$ being the ion sound speed and gyrofrequency) accompanied by features of plasma energization were observed on the auroral and cusp field lines (see, e.g., Stasiewicz et al. 2000, and references therein). These structures were referred to as solitary KAWs because of their large amplitude and localization (Louarn et al. 1994; Wahlund et al. 1994).

Most of the work related to filamentation is done in the framework of the Hall MHD (Champeaux et al. 1997, 1998). However, not much of the analytical or numerical work has been done on the KAW filamentation. In this paper we have studied the filamentation of a perturbation present on a KAW (we term it the main KAW) in the presence of the second KAW. The paper is organized as follows. In Sect. 2, the beam width parameter of the main KAW as well as that of perturbation is derived in the presence of the second KAW in the intermediate $\beta$ regime $\left(m_{\mathrm{e}} / m_{\mathrm{i}} \ll \beta \ll 1\right)$. The threshold (critical) field values of the main KAW and the perturbation present on it are also obtained. This work has been applied to the solar wind. The third section provides discussion and conclusion.

\section{Model equations}

Consider two linearly polarized Kinetic Alfvén waves (KAW) propagating in $x-z$ plane of the magnetized plasma having ambient magnetic field B along the $z$ axis. The initial $(z=0)$ magnetic field intensity distribution of the KAWs without perturbation is assumed to be of the form

$\left.\tilde{B}_{10,20} \tilde{B}_{10,20}^{*}\right|_{z=0}=\tilde{B}_{100,200}^{2} \exp \left(\frac{-x^{2}}{r_{10,20}^{2}}\right)$.

To avoid repeating the description of the two KAWs, we have represented them in a single equation. Therefore the symbol $B_{100,200}$ stands for $B_{100}$ and $B_{200}$. Similarly $B_{10,20}$ stands for $B_{10}$ and $B_{20}$. Here $r_{10}$ and $r_{20}$ are the transverse scale size of the two KAWs and $\tilde{B}_{100}$ and $\tilde{B}_{200}$ are their magnetic field amplitudes at $z=0$ and $x=0$. $\tilde{B}_{10}$ and $\tilde{B}_{20}$ are the magnetic field amplitudes of the two KAWs at any $x$ and $z$. Let a perturbation be superimposed on the first KAW such that initial magnetic field intensity distribution of the perturbation is given by

$\left.\tilde{B}_{11} \tilde{B}_{11}^{*}\right|_{z=0}=\tilde{B}_{110}^{2} \exp \left(\frac{-x^{2}}{r_{110}^{2}}\right)$

where $r_{110}$ is the transverse scale size of the perturbation and $\tilde{B}_{110}$ is the magnetic field amplitude of the perturbation at $z=0$ and $x=0$. The total magnetic field vector of the main KAW wave $\left(\tilde{B}_{1}\right)$ with perturbation can be written as

$\tilde{B}_{1}=\tilde{B}_{10}+\tilde{B}_{11}$.

Here $\tilde{B}_{10}$ is the magnetic field of the main KAW without perturbation and $\tilde{B}_{11}$ is the magnetic field of the perturbation superimposed on it.

The dynamical equation governing the propagation of the KAW in the $x-z$ plane can be obtained by using the standard method (Bellan et al. 1998; Shukla \& Stenflu 1999, 2000; Shukla \& Sharma 2002; Shukla \& Sharma et al. 2004) and can be written as

$\frac{\partial^{2} B_{1,2}}{\partial t^{2}}=-V_{\mathrm{Te}}^{2} \lambda_{\mathrm{e}}^{2} \frac{\partial^{4} B_{1,2}}{\partial x^{2} \partial z^{2}}+v_{\mathrm{A}}^{2}\left(1-\frac{\delta n_{\mathrm{s}}}{n_{0}}\right) \frac{\partial^{2} B_{1,2}}{\partial z^{2}}$.
Here $B_{1,2}$ indicates the $y$ component of the total magnetic field $B_{1}$ and $B_{2}$ of the KAWs separately. $n_{0}$ is the electron density of plasma in the absence of waves and $\delta n_{\mathrm{s}}$ is the number density change given by $n_{\mathrm{e}}-n_{0}$, with $n_{\mathrm{e}}$ being the modified electron density. The Eq. (4) of the KAW has been derived from fluid equations when $1 \gg \beta \gg m_{\mathrm{e}} / m_{\mathrm{i}}$. In this $\beta$ limit, the dispersion relation of the KAW is the same as that obtained by Gekelman from the expansion of plasma dispersion function (Vlasov approach $)$ in the limit of $\left(\omega \ll k_{z} V_{\mathrm{Te}}\right)$. The density can be modified due to both the nonlinear electron heating driven nonlinearity and the ponderomotive force by the KAW. Also,

$V_{\mathrm{Te}}\left(=\sqrt{\frac{T_{\mathrm{e}}}{m_{\mathrm{e}}}}\right)$ is the electron thermal speed,

$v_{\mathrm{A}}\left(=\sqrt{\frac{B^{2}}{4 \pi n_{0} m_{\mathrm{i}}}}\right)$ is the Alfvén speed

and $\lambda_{\mathrm{e}}\left(=\sqrt{\frac{c^{2} m_{\mathrm{e}}}{4 \pi n_{0} e^{2}}}\right)$ is the electron inertia length.

Consider a plane wave solution of Eq. (4) as

$B_{1,2}=\tilde{B}_{1,2}(x, z) \mathrm{e}^{\mathrm{i}\left(k_{x 1,2} x+k_{z_{1,2}} z-\omega_{1,2} t\right)}$.

Using Eqs. (4) and (5), one gets

$-2 \mathrm{i} k_{z_{1,2}} \frac{\partial \tilde{B}_{1,2}}{\partial z}-V_{\mathrm{Te}}^{2} \lambda_{\mathrm{e}}^{2} k_{z_{1,2}}^{2} \frac{\partial^{2} \tilde{B}_{1,2}}{\partial x^{2}}-k_{z_{1,2}}^{2} \frac{\partial n_{\mathrm{s}}}{n_{0}} \tilde{B}_{1,2}=0$

Here, $\omega_{1,2}$ is the frequency of the KAW.

Also,

$\frac{\partial \tilde{B}_{1,2}}{\partial z} \ll k_{z 1,2} \tilde{B}_{1,2}$ and $\frac{\partial \tilde{B}_{1,2}}{\partial x} \gg k_{x_{1,2}} \tilde{B}_{1,2}$

and

$\frac{\delta n_{\mathrm{s}}}{n_{0}}=\phi\left(\tilde{B}_{1} \tilde{B}_{1}^{*}, \tilde{B}_{2} \tilde{B}_{2}^{*}\right)$.

Here, $\phi\left(\tilde{B}_{1} \tilde{B}_{1}^{*}, \tilde{B}_{2} \tilde{B}_{2}^{*}\right)$ for $m_{\mathrm{e}} / m_{\mathrm{i}} \ll \beta \ll 1$ can be written as (Bellan et al. 1998; Shukla et al. 1999, 2000; Shukla \& Sharma 2002; Shukla et al. 2004)

$\phi\left(\tilde{B}_{1} \tilde{B}_{1}^{*}, \tilde{B}_{2} \tilde{B}_{2}^{*}\right)=\exp \left(\gamma_{1} \tilde{B}_{1} \tilde{B}_{1}^{*}+\gamma_{2} \tilde{B}_{2} \tilde{B}_{2}^{*}\right)-1$.

Here,

$\gamma_{1,2}=\frac{1-\alpha_{0_{1,2}}\left(1+\delta_{1,2}\right)}{16 \pi n_{0} T} \frac{v_{\mathrm{A}}^{2} k_{z_{1,2}}}{\omega_{1,2}^{2}}$

$\alpha_{0_{1,2}}=\frac{\omega_{1,2}^{2}}{\omega_{\mathrm{ci}}^{2}}$

$\delta_{1,2}=\frac{m_{\mathrm{e}}}{m_{\mathrm{i}}}\left(\frac{k_{x_{1,2}}}{k_{z_{1,2}}}\right)^{2}$.

Using Eq. (6), the field vector of the main $\operatorname{KAW}\left(\tilde{B}_{10,20}\right)$ and the perturbation $\left(\tilde{B}_{11}\right)$ satisfy the following equations:

$$
\begin{array}{r}
-2 \mathrm{i} k_{z_{1,2}} \frac{\partial \tilde{B}_{10,20}}{\partial z}-\frac{1}{v_{\mathrm{A}}^{2}}\left(V_{\mathrm{Te}}^{2} \lambda_{\mathrm{e}}^{2} k_{z_{1,2}}^{2}\right) \frac{\partial^{2} \tilde{B}_{10,2}}{\partial x^{2}} \\
-k_{z_{1,2}}^{2} \phi\left(\tilde{B}_{1} \tilde{B}_{1}^{*}, \tilde{B}_{2} \tilde{B}_{2}^{*}\right) \tilde{B}_{10,20}=0
\end{array}
$$


and

$$
\begin{aligned}
-2 \mathrm{i} k_{z_{1}} & \frac{\partial \tilde{B}_{11}}{\partial z}-\frac{1}{v_{\mathrm{A}}^{2}}\left(V_{\mathrm{Te}}^{2} \lambda_{\mathrm{e}}^{2} k_{z_{1}}^{2}\right) \frac{\partial^{2} \tilde{B}_{11}}{\partial x^{2}} \\
& -k_{z_{1}}^{2}\left\{\phi\left(\tilde{B}_{1} \tilde{B}_{1}^{*}, \tilde{B}_{2} \tilde{B}_{2}^{*}\right)-\phi\left(\tilde{B}_{10} \tilde{B}_{10}^{*}, \tilde{B}_{2} \tilde{B}_{2}^{*}\right)\right\} \tilde{B}_{10} \\
& -k_{z_{1}}^{2} \phi\left(\tilde{B}_{1} \tilde{B}_{1}^{*}, \tilde{B}_{2} \tilde{B}_{2}^{*}\right) \tilde{B}_{11}=0 .
\end{aligned}
$$

Following Akhmanov et al. (1968) and Shukla et al. (2002), the solution for $B_{10,20}$ can be written as:

$$
\begin{aligned}
& \tilde{B}_{10,20}=\tilde{A}_{10,20} \exp \left(\mathrm{i} k_{z_{10,20}} S_{10,20}(x, z)\right) \\
& \tilde{A}_{10,20}^{2}=\frac{\tilde{B}_{100,200}^{2}}{f_{10,20}} \exp \left(\frac{-x^{2}}{r_{10,20}^{2} f_{10,20}^{2}}\right) \\
& S_{10,20}=\beta_{10,20} \frac{x^{2}}{2}+\phi_{10,20} \\
& \beta_{10,20}=a_{1,2} \frac{1}{f_{10,20}} \frac{\mathrm{d} f_{10,20}}{\mathrm{~d} z} \\
& a_{1,2}=-\frac{v_{\mathrm{A}}^{2}}{V_{\mathrm{Te}}^{2} \lambda_{\mathrm{e}}^{2} k_{z_{1,2}}^{2}} .
\end{aligned}
$$

In Eq. (10), $f_{10,20}$ are the dimensionless beam width parameters of the two KAWs and it are governed by the differential equation:

$$
\begin{aligned}
\frac{\mathrm{d}^{2} f_{10,20}}{\mathrm{~d} z^{2}}= & \frac{1}{a_{1,2}^{2} k_{z_{1,2}}^{2} r_{10,20}^{2} f_{10,20}^{3}}-\frac{f_{10,20}}{a_{1,2}}\left(\frac{\gamma_{1} B_{100}^{2}}{r_{10}^{2} f_{10}^{3}}+\frac{\gamma_{2} B_{200}^{2}}{r_{20}^{2} f_{20}^{3}}\right) \\
& \times \exp \left(\frac{\gamma_{1} \tilde{B}_{100}^{2}}{f_{10}}+\frac{\gamma_{2} \tilde{B}_{200}^{2}}{f_{20}}\right) .
\end{aligned}
$$

The condition for the self trapping mode for the KAW is obtained by balancing the two terms on the right side of Eq. (11) at $z=0$, given by

$$
\left(\frac{\gamma_{1} B_{100}^{2}}{r_{10}^{2}}+\frac{\gamma_{2} B_{200}^{2}}{r_{20}^{2}}\right) \exp \left(\gamma_{1} \tilde{B}_{100}^{2}+\gamma_{2} \tilde{B}_{200}^{2}\right)=\frac{1}{a_{1,2} k_{z_{1,2}}^{2} r_{10,20}^{2}} .
$$

The initial conditions on $f_{10,20}$ for the plain wave front are:

$$
\frac{\mathrm{d} f_{10,20}}{\mathrm{~d} z}=0 \text { and } f_{10,20}=1 \text { at } z=0 \text {. }
$$

To obtain the solution of Eq. (9), we express the perturbation field as

$\tilde{B}_{11}=\tilde{A}_{11}(x, z) \exp \left(\iota k_{z_{1}} S_{11}(x, z)\right)$

where $S_{11}$ is the eikonal (i.e. representing the change in the phase due to non-linear effects). Substituting for $\tilde{B}_{11}$ from Eq. (13) into Eq. (9), we obtain following equations after separating real and imaginary parts:

$2 \frac{\partial S_{11}}{\partial z}-\frac{V_{\mathrm{Te}}^{2} \lambda_{\mathrm{e}}^{2} k_{z_{1}}^{2}}{v_{\mathrm{A}}^{2}}\left(\frac{\partial S_{11}}{\partial x}\right)^{2}=-\frac{V_{\mathrm{Te}}^{2} \lambda_{\mathrm{e}}^{2}}{v_{A}^{2} \tilde{A}_{11}} \frac{\partial^{2} \tilde{A}_{11}}{\partial x^{2}}+\phi_{\mathrm{eff}}$

where

$$
\begin{aligned}
\phi_{\mathrm{eff}}= & \phi\left(\tilde{B}_{1} \tilde{B}_{1}^{*}, \tilde{B}_{2} \tilde{B}_{2}^{*}\right) \\
& +\cos \left(\phi_{\mathrm{p}}\right) \frac{\tilde{A}_{10}}{\tilde{A}_{11}}\left[\phi\left(\tilde{B}_{1} \tilde{B}_{1}^{*}, \tilde{B}_{2} \tilde{B}_{2}^{*}\right)-\phi\left(\tilde{B}_{10} \tilde{B}_{10}^{*}, \tilde{B}_{2} \tilde{B}_{2}^{*}\right)\right]
\end{aligned}
$$

and

$$
\begin{aligned}
& \frac{\partial \tilde{A}_{11}^{2}}{\partial z}-\frac{1}{v_{A}^{2}}\left(V_{\mathrm{Te}}^{2} \lambda_{\mathrm{e}}^{2} k_{z_{1}}^{2}\right)\left(\frac{\partial \tilde{A}_{11}^{2}}{\partial x} \frac{\partial S_{11}}{\partial x}+\tilde{A}_{11}^{2} \frac{\partial^{2} S_{11}}{\partial x^{2}}\right) \\
& +\left[\gamma_{1} k_{z_{1}} \tilde{A}_{10}^{2} \sin \left(2 \phi_{\mathrm{p}}\right) \exp \left(\gamma_{1} \tilde{B}_{10} \tilde{B}_{10}^{*}+\gamma_{2} \tilde{B}_{2} \tilde{B}_{2}^{*}\right)\right] \tilde{A}_{11}^{2}=0 .
\end{aligned}
$$

Here, $\phi_{\mathrm{p}}=-k_{z 1}\left(S_{11}-S_{10}\right)$. $\phi_{\text {eff }}$ represents the modification in dimensionless density when the non-linear interaction between the main KAW and the perturbation present on it has been taken in to account.

$\phi_{\mathrm{p}}$ is the measure of the change in the angle between the magnetic field of the main wave and that of the perturbation. This angle changes with the distance of propagation because of the nonlinear effects. The value of $\phi_{\mathrm{p}}$ depends on the phase angle between the main KAW field and that of the perturbation field at $z=0$ and the phase shift introduced in the perturbation field due to the nonlinearity in the medium.

In writing Eq. (14b) the nonlinear part has been expanded as

$$
\begin{aligned}
\phi\left(\tilde{B}_{1} \tilde{B}_{1}^{*}, \tilde{B}_{2} \tilde{B}_{2}^{*}\right)= & \phi\left(\tilde{B}_{10} \tilde{B}_{10}^{*}, \tilde{B}_{2} \tilde{B}_{2}^{*}\right) \\
& +\left.\frac{\partial \phi}{\partial \tilde{B}_{1} \tilde{B}_{1}^{*}}\right|_{\tilde{B}_{1} \tilde{B}_{1}^{*}=\tilde{B}_{10} \tilde{B}_{10}^{*}}\left(\tilde{B}_{1} \tilde{B}_{1}^{*}-\tilde{B}_{10} \tilde{B}_{10}^{*}\right) .
\end{aligned}
$$

Following Akhmanov et al. (1968), the solution of Eqs. (14a) and (14b) can be written as

$\tilde{A}_{11}=\frac{\tilde{B}_{110}}{f_{11}^{1 / 2}} \mathrm{e}^{\left(-x^{2} / 2 r_{11}^{2} f_{11}^{2}\right)} \mathrm{e}^{-k_{i} z}$

Also

$S_{11}=\frac{x^{2}}{2} \beta_{11}(z)+\phi_{11}(z)$

where $\beta_{11}=a \frac{1}{f_{11}} \frac{\mathrm{d} f_{11}}{\mathrm{~d} z}$.

Where $f_{11}$ is the dimensionless beam width parameter of the perturbation, the inverse of $\beta_{11}$ represent radius of curvature of the wavefront. The growth rate $k_{i}$ is given by

$k_{i}=\frac{1}{2} k_{z_{1}} \gamma_{1} \tilde{A}_{10}^{2} \sin \left(2 \phi_{\mathrm{p}}\right) \mathrm{e}^{\left(\gamma_{1} \tilde{\mathrm{A}}_{10}^{2}+\gamma_{2} \tilde{\mathrm{A}}_{2}^{2}\right)}$

and

$a_{1}=-\frac{v_{\mathrm{A}}^{2}}{V_{\mathrm{Te}}^{2} \lambda_{\mathrm{e}}^{2} k_{z_{1}}^{2}}$.

Using Eqs. (15) to (20) in Eq. (14a), we obtain the following equation for $f_{11}$, after equating coefficients of $x^{2}$ in Eq. (14a):

$\frac{\mathrm{d}^{2} f_{11}}{\mathrm{~d} \xi^{2}}=\frac{1}{a_{1}^{2} k_{z_{1}}^{2} r_{11}^{4}} \frac{1}{f_{11}^{3}}-\frac{f_{11}}{a_{1}} \Phi$

where

$$
\begin{aligned}
\Phi & =\left.\left(\frac{\gamma_{1} \tilde{B}_{100}^{2}}{r_{10}^{2} f_{10}^{3}}+\frac{\gamma_{2} \tilde{B}_{200}^{2}}{r_{20}^{2} f_{20}^{3}}\right) \mathrm{e}^{\left(\frac{\gamma_{1} \tilde{B}_{100}^{2}}{f_{1}}+\frac{\gamma_{2} \tilde{B}_{200}^{2}}{f_{2}}\right)}\right|_{x^{2}=0} \\
& -\mathrm{e}^{\left(\frac{\gamma_{1} \tilde{E}_{100}^{2}}{f_{1}}+\frac{\gamma_{2} \tilde{B}_{200}^{2}}{f_{2}}\right)}\left(\frac{\gamma_{1} \tilde{B}_{100}^{2}}{r_{10}^{2} f_{10}^{3}}+\frac{\gamma_{2} \tilde{B}_{200}^{2}}{r_{20}^{2} f_{20}^{3}}\right) \\
& \left\{3 \gamma_{1} \cos \left(\phi_{\mathrm{p}}\right)\left(\frac{1}{f_{11} f_{10}}\right)^{1 / 2} \tilde{B}_{100} \tilde{B}_{110} \mathrm{e}^{\left(-k_{i} z\right)}+\frac{2 \gamma_{1} \tilde{B}_{100}^{2} \cos ^{2}\left(\phi_{\mathrm{p}}\right)}{f_{10}}\right\} \\
& +\mathrm{e}^{\left(\frac{\gamma_{1} \tilde{B}_{100}^{2}}{f_{1}}+\frac{\gamma_{2} \tilde{B}_{200}^{2}}{f_{2}}\right)}\left\{\begin{array}{l}
3 \gamma_{1} \cos \left(\phi_{\mathrm{p}}\right) \tilde{B}_{100} \tilde{B}_{110}\left(\frac{1}{f_{11} f_{10}}\right)^{1 / 2} \mathrm{e}^{\left(-k_{i} z / 2\right)} \\
\left(\frac{1}{2 r_{11}^{2} f_{11}^{2}}+\frac{1}{2 r_{11}^{2} f_{11}^{2}}\right)+\frac{3 \gamma_{1} \tilde{B}_{100}^{2} \cos ^{2}\left(\phi_{\mathrm{p}}\right)}{2 r_{10}^{2} f_{10}^{3}}
\end{array}\right\} .(22)
\end{aligned}
$$

Equation (21) determines the convergence/divergence of the perturbation. The above solution has been obtained in the 
paraxial approximation $\left(x^{2} \ll r_{10,2}^{2} f_{1,2}^{2}\right)$. The critical field of the perturbation for self-trapping is obtained by balancing two terms in the right side of Eq. (21). The corresponding condition for the critical magnetic field is given by

$$
\begin{aligned}
& 3 \gamma_{1} B_{110} B_{100} \cos \left(\phi_{\mathrm{p}}\right) \mathrm{e}^{\left(\gamma_{1} \tilde{B}_{100}^{2}+\gamma_{2} \tilde{B}_{200}^{2}\right)} \\
& \times\left[-\left(\frac{\gamma_{1} \tilde{B}_{100}^{2}}{r_{10}^{2}}+\frac{\gamma_{2} \tilde{B}_{200}^{2}}{r_{20}^{2}}\right)-\frac{1}{2 r_{10}^{2}}-\frac{1}{2 r_{11}^{2}}\right]= \\
& \frac{1}{a_{11} r_{11}^{4} k_{z_{1}}^{2}}-\left(\frac{\gamma_{1} \tilde{B}_{100}^{2}}{r_{10}^{2}}+\frac{\lambda_{2} \tilde{B}_{200}^{2}}{r_{20}^{2}}\right) \mathrm{e}^{\left(\gamma_{1} \tilde{B}_{100}^{2}+\gamma_{2} \tilde{B}_{200}^{2}\right)} \\
& -\mathrm{e}^{\left(\gamma_{1} \tilde{B}_{100}^{2}+\gamma_{2} \tilde{B}_{200}^{2}\right)}\left[\begin{array}{l}
\left.\left(\frac{\gamma_{1} \tilde{B}_{100}^{2}}{r_{10}^{2}}+\frac{\gamma_{2} \tilde{B}_{200}^{2}}{r_{20}^{2}}\right) 2 \gamma_{1} \tilde{B}_{100}^{2} \cos ^{2}\left(\phi_{\mathrm{p}}\right)\right] . \\
+\frac{2 \gamma_{1} \tilde{B}_{100}^{2} \cos ^{2}\left(\phi_{\mathrm{p}}\right)}{r_{10}^{2}}
\end{array}\right] .
\end{aligned}
$$

Using the parameters given below, the critical fields for the main KAWs and the perturbation can be calculated by making use of the equations defined above. Now we would like to make an estimate of these critical fields for the main KAWs and the perturbation for the solar wind.

The typical solar wind parameters (Jokipii 1973; Dendy 1995 ) for $\beta=0.121$ are as given below (around $1 \mathrm{AU}$ ).

$T_{\mathrm{e}}=0.5 \times 10^{5} \mathrm{~K} ; T_{\mathrm{i}}=0.2 \times 10^{5} \mathrm{~K} ; B_{0}=1 \times 10^{-4} \mathrm{G}$; $f_{\mathrm{pe}}=20 \times 10^{3} \mathrm{~Hz}$; number of protons $=5$ protons $/$ c.c.

We find that

$f_{\mathrm{ci}}=0.152 \mathrm{~Hz} ; \rho=2.5 \times 10^{6} \mathrm{~cm} ; v_{\mathrm{A}}=9.8 \times 10^{6} \mathrm{~cm} / \mathrm{s} ; V_{\mathrm{Te}}=$ $8.7 \times 10^{7} \mathrm{~cm} / \mathrm{s}$.

For $\omega_{1}=0.04 \mathrm{~Hz}$ and $k_{x 1} \rho=0.01$; we get $k_{z 1}=0.41 \times$ $10^{-8} \mathrm{~cm}^{-1} ; k_{x 1}=0.4 \times 10^{-8} \mathrm{~cm}^{-1}$.

For $\omega_{2}=0.06 \mathrm{~Hz}$ and $k_{x 2} \rho=0.01$; we get $k_{z 2}=0.62 \times$ $10^{-8} \mathrm{~cm}^{-1} ; k_{x 2}=0.4 \times 10^{-8} \mathrm{~cm}^{-1}$.

Here $f_{\mathrm{ci}}$ is the ion cyclotron frequency and $f_{\mathrm{pe}}$ is the electron plasma frequency.

For these typical parameters, the critical value of the magnetic field as given by Eq. (12) is $3.24 \times 10^{-8} \mathrm{G}\left(\eta_{1}=3.24 \times 10^{-4}\right.$ for $\eta_{2}=0$ ) for $r_{10}=7.66 \times 10^{3} \mathrm{~km}$ and $2.67 \times 10^{-8} \mathrm{G}$ $\left(\eta_{2}=2.67 \times 10^{-4}\right.$ for $\left.\eta_{1}=0\right)$ for $r_{20}=5.10 \times 10^{3} \mathrm{~km}$ (here $\left.\eta_{1,2}=B_{y_{100,200}} / B_{0}\right)$

The critical magnetic field value of the perturbation as given by Eq. (23) is $8.6939 \times 10^{-12} \mathrm{G}$, for the main kinetic Alfvén wave field $3.24 \times 10^{-8} \mathrm{G}, r_{10}=7.66 \times 10^{3} \mathrm{~km}$ and the other KAW field $2.67 \times 10^{-8} \mathrm{G}, 5.10 \times 10^{3} \mathrm{~km}$ and $r_{110}=4.54 \times 10^{3} \mathrm{~km}$.

\section{Discussion and conclusion}

We study the effect of the mutual nonlinear interaction of the two kinetic Alfvén waves on the (their) filament formation. The initial wavefront of the KAWs are assumed to be Gaussian. Theses studies have been done in the paraxial approximations $\left(x \ll r_{10} f_{1}\right)$. We have investigated the dependence of the filamentation of the main (first) KAW on the initial magnetic field strength, and the frequency of the second KAW for the typical parameters of the solar wind. When the two KAWs are present the background density modification depends on the intensity of both the kinetic Alfvén waves as given by Eq. (7). Therefore, the non-linear term governing the beam width parameter will be modified accordingly in comparison with a single KAW case.

Figures 1a,b depict the intensity distribution of the main KAW in the solar wind at different distances along the direction of propagation but at different $x$ values. The filamentary structures are seen at different locations. This can be explained using Eq. (11). When the initial magnetic field (maximum field at

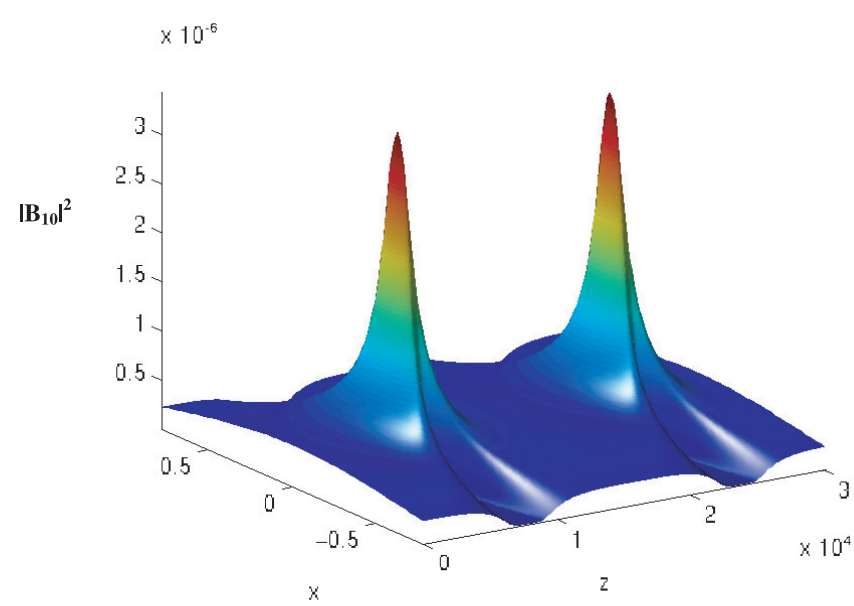

(a)

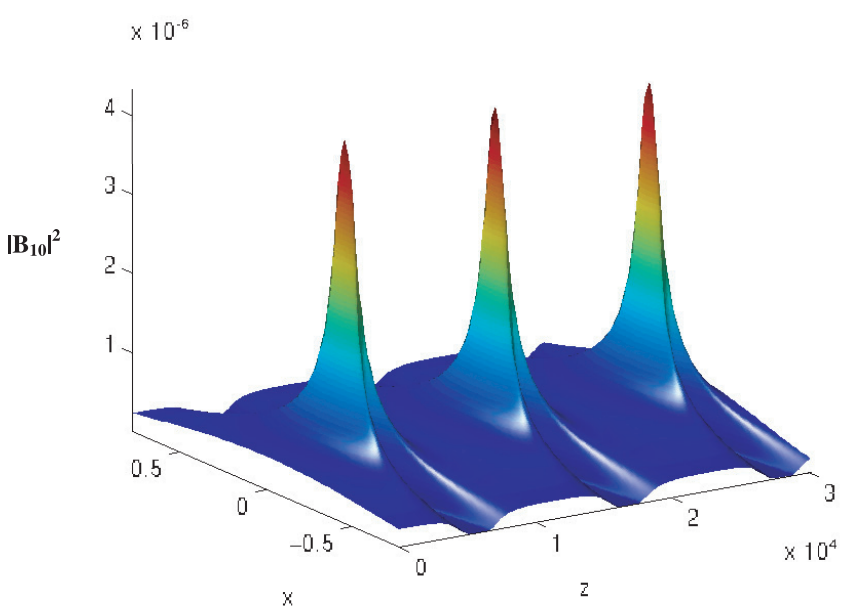

(b)

Fig. 1. a) The variation of the magnetic field intensity of the Main KAW for $f_{1}=0.04 \mathrm{~Hz}, f_{2}=0.06 \mathrm{~Hz}, B_{100}(z=0, x=0)=3.235 \times 10^{-8} \mathrm{G}$, $B_{200}(z=0, x=0)=2.915 \times 10^{-8} \mathrm{G}$ and $r_{10}=76.5 \times 10^{3} \mathrm{~km}, r_{20}=$ $51.1 \times 10^{3} \mathrm{~km}$. b) The variation of the magnetic field intensity of the Main KAW for $f_{1}=0.04 \mathrm{~Hz}, f_{2}=0.06 \mathrm{~Hz}, B_{100}(z=0, x=0)=3.235 \times$ $10^{-8} \mathrm{G}, B_{200}(z=0, x=0)=4.858 \times 10^{-8} \mathrm{G}$ and $r_{10}=76.5 \times 10^{3} \mathrm{~km}$, $r_{20}=51.1 \times 10^{3} \mathrm{~km}$.

$z=0$ ) of the KAW is more than its critical magnetic field, the non-linear term (second term in the right hand side of Eq. (11)) dominates and the value of $f_{10}$ decreases with the distance of propagation. But when $f_{10}$ becomes very small, the diffraction term (first term in the right hand side of the Eq. (11)) starts to dominate. Therefore, $f_{10}$ increases with the distance of the propagation until $f_{10}$ become so large that the diffraction term becomes smaller compared to the nonlinear term. $f_{10}$ further decreases due to the nonlinear effects, until it becomes so small that the diffraction term again dominates and $f_{10}$ start to diverge and this process repeats. Hence, the main KAW attains a certain minimum beam width parameter $\left(f_{10}\right)$, and the intensity of the main KAW in these small size structures becomes very high.

Figures $1 \mathrm{a}$ and $\mathrm{b}$ display the variation of the intensity of the main KAW for different values of the initial magnetic field strength of the second KAW in the solar wind. In the presence of the second KAW, the intensity profile of the filaments are greatly modified. This is because the additional term arising due to the 


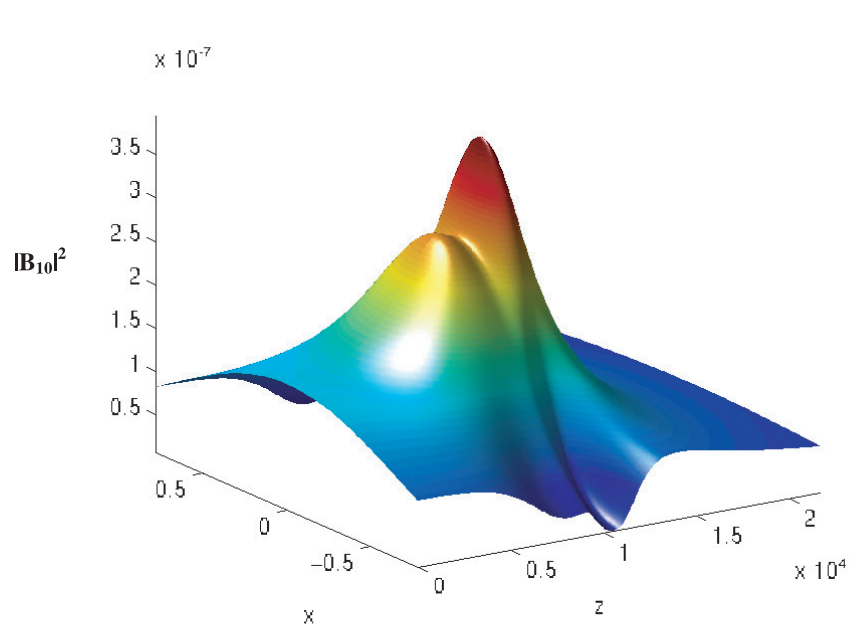

(a)

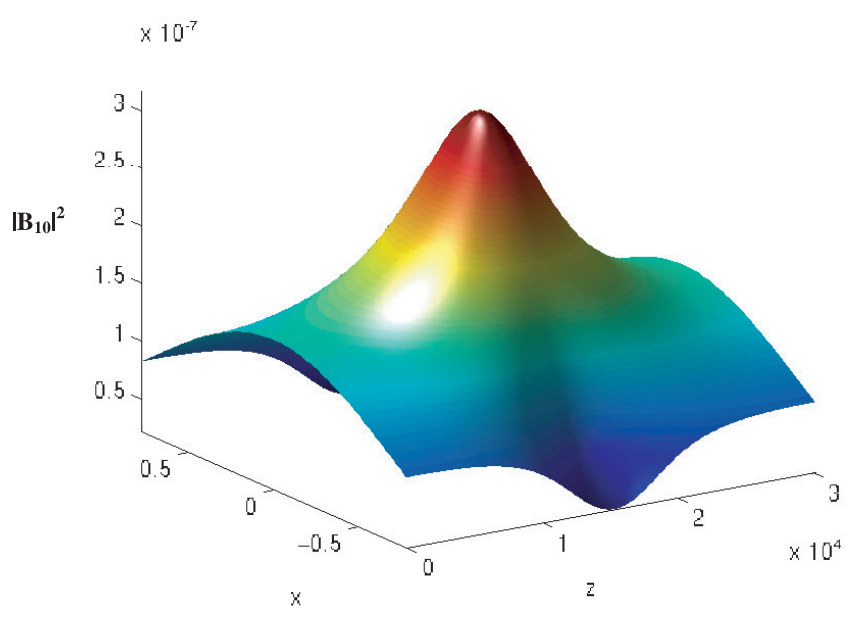

(b)

Fig. 2. a) The variation of the magnetic field intensity of the Main KAW for $f_{1}=0.04 \mathrm{~Hz}, f_{2}=0.02 \mathrm{~Hz}, B_{100}(z=0, x=0)=1.941 \times 10^{-8} \mathrm{G}$, $B_{200}(z=0, x=0)=0.582 \times 10^{-8} \mathrm{G}$ and $r_{10}=76.5 \times 10^{3} \mathrm{~km}, r_{20} f=$ $15.3 \times 10^{4} \mathrm{~km}$. b) The variation of the magnetic field intensity of the Main KAW for $f_{1}=0.04 \mathrm{~Hz}, f_{2}=0.02 \mathrm{~Hz}, B_{100}(z=0, x=0)=1.941 \times$ $10^{-8} \mathrm{G}, B_{200}(z=0, x=0)=1.165 \times 10^{-8} \mathrm{G}$ and $r_{10}=76.5 \times 10^{3} \mathrm{~km}$, $r_{20}=76.5 \times 10^{3} \mathrm{~km}$.

presence of the second KAW in the nonlinear term of Eq. (11) will change the rate of change of the non-linear term with $z$ and hence the balance of the nonlinear term and the diffraction term takes place at a lower value of $f$. This effect is equivalent to the case if we increase the intensity of the main (first) KAW.

Next we studied the effect of changing the frequency of the second KAW on the filament formation of the main KAW. Figures $2 \mathrm{a}, \mathrm{b}$ display the intensity profile of the filaments of the main KAW for different frequencies. When we increase the frequency of the second KAW, the intensity of the filaments of the main KAW decreases and the spacing between them increases. This happens because of the complex nonlinear dynamics of the beam width of the main beam field on the frequency

Next we have studied the effect of the presence of the second KAW on a perturbation present on the main KAW. First we studied the dependence of the filamentation of the perturbation by changing the frequency of the second KAW. Figures $3 a, b$ show the intensity of the perturbation for different frequencies of the

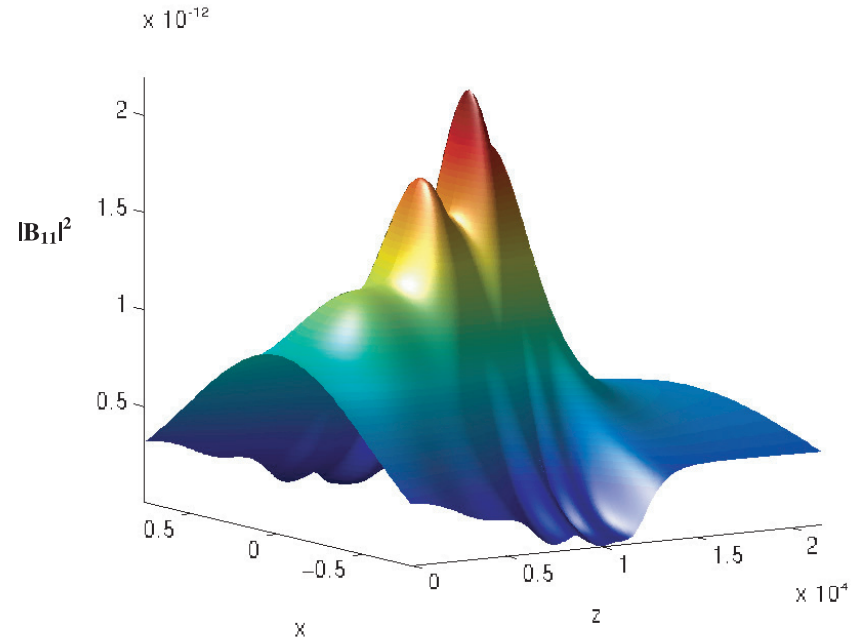

(a)

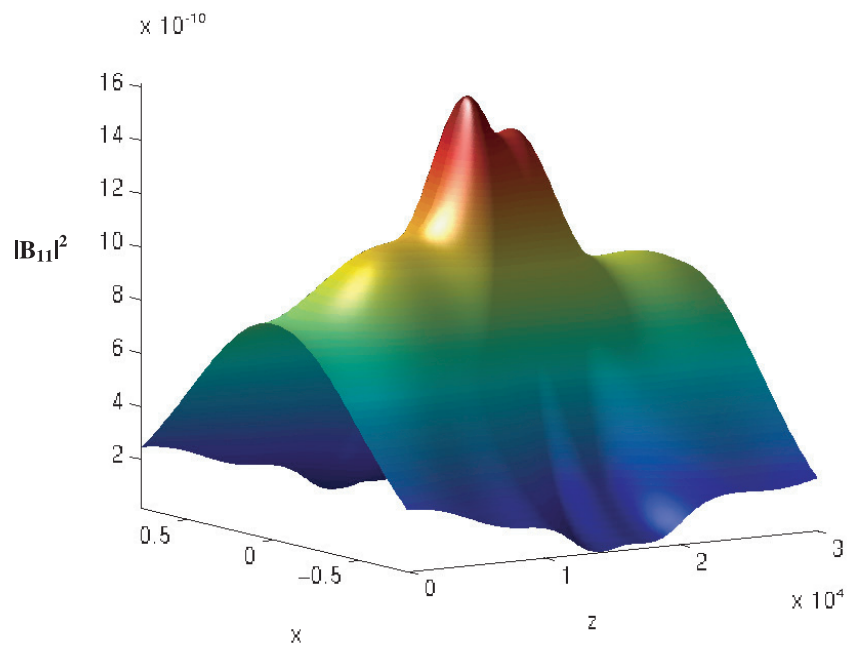

(b)

Fig. 3. a) The magnetic field intensity of the perturbation present on the main KAW for $f_{1}=0.04 \mathrm{~Hz}, f_{2}=0.02 \mathrm{~Hz}, B_{100}(z=0, x=0)=1.941 \times$ $10^{-8} \mathrm{G}, B_{200}(z=0, x=0)=0.582 \times 10^{-8} \mathrm{G}$ and $r_{10}=76.5 \times 10^{3} \mathrm{~km}$, $r_{20}=15.3 \times 10^{4} \mathrm{~km}$. b) The magnetic field intensity of the perturbation present on the main KAW for $f_{1}=0.04 \mathrm{~Hz}, f_{2}=0.04 \mathrm{~Hz}, B_{100}(z=0$, $x=0)=1.941 \times 10^{-8} \mathrm{G}, B_{200}(z=0, x=0)=1.165 \times 10^{-8} \mathrm{G}$ and $r_{10}=76.5 \times 10^{3} \mathrm{~km}, r_{20}=76.5 \times 10^{3} \mathrm{~km}$.

second KAW. The increases in the frequency of the second KAW result in decrease in magnetic field intensity of the filaments of the perturbation and the spacing between the primary filaments increases.

Figures $4 a, b$ illustrates the dependence of the intensity of the perturbation on the magnetic field intensity of the second wave. The increases in the magnetic field intensity of the second KAW results in the increase in the magnetic field intensity of the filaments of the perturbation. The abovementioned process, filamentation, is very important in transferring the energy of KAWs from large scale (smaller frequency) to small scale (higher frequency) so that Landau damping is possible. Because of the filamentation, higher modes appear and the energy that was localized in the lower modes will be redistributed in the higher modes. This will change the spectrum of the Alfvénic turbulence in the solar wind. Due to this, the spectral index is also expected to change. The presence of secondary filaments in the intensity 


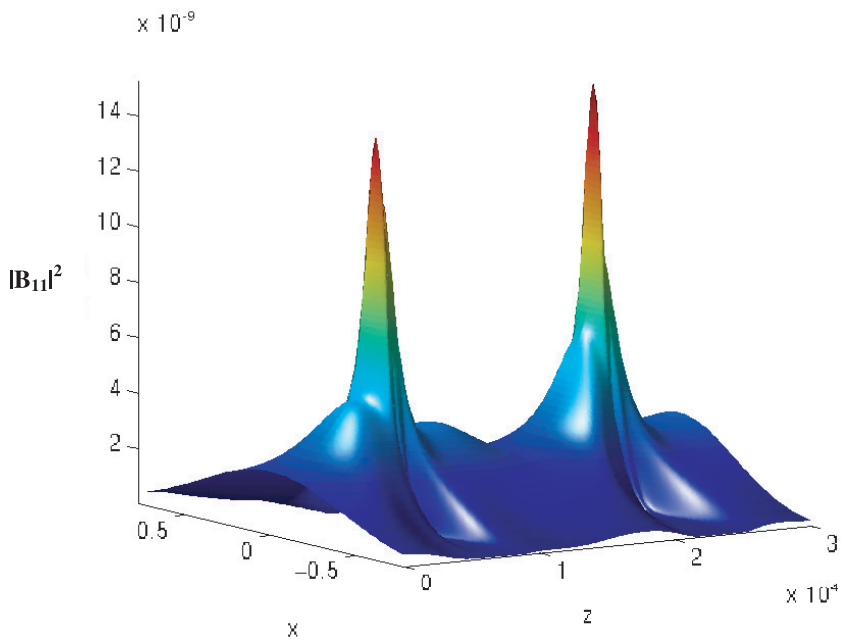

(a)

$\times 10^{-9}$

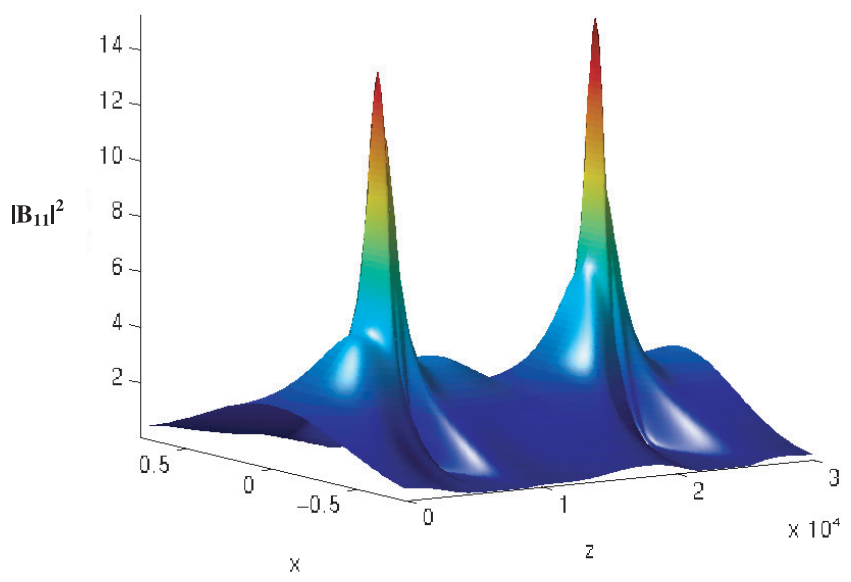

(b)

Fig. 4. a) The magnetic field intensity of the perturbation present on the main KAW for $f_{1}=0.04 \mathrm{~Hz}, f_{2}=0.06 \mathrm{~Hz}, B_{100}(z=0, x=0)=3.235 \times$ $10^{-8} \mathrm{G}, B_{200}(z=0, x=0)=4.858 \times 10^{-8} \mathrm{G}$ and $r_{10}=76.5 \times 10^{3} \mathrm{~km}$, $r_{20}=51.1 \times 10^{3} \mathrm{~km}$. b) The magnetic field intensity of the perturbation present on the main KAW for $f_{1}=0.04 \mathrm{~Hz}, f_{2}=0.06 \mathrm{~Hz}, B_{100}(z=0$, $x=0)=3.235 \times 10^{-8} \mathrm{G}, B_{200}(z=0, x=0)=4.858 \times 10^{-8} \mathrm{G}$ and $r_{10}=76.5 \times 10^{3} \mathrm{~km}, r_{20}=51.1 \times 10^{3} \mathrm{~km}$.

profile of the perturbation present on the main KAW will further affect the spectral transfer of the energy from larger scale to smaller scale. The relevance of this to heating and acceleration of the solar wind, coronal heating and the auroral formation can also be extended. The heating rate can be estimated by using the velocity space diffusion coefficient and the Fokker Planck equation or from more rigorous models. In order to study these effects we need to study the effect of filament formation on the wavenumber spectrum. In Fig. 5 we have presented these studies in one typical case when the filaments are formed by the nonlinear interaction of two KAWs without perturbation.

We studied the Fourier components $\left|B_{k}\right|^{2}$ for the main KAW against $k$ at a fixed $x$ value in Fig. 5. Figure 5 is obtained from Fig. $1 \mathrm{~b}$ of the filamentary structures. The data set was obtained at $x=0$ and the FFT was taken and $\left|B_{k}\right|^{2}$ against $k_{z}$ was plotted. This figure depicts the power spectra $\left|B_{k}\right|^{2}$ against $k$ at fixed $x$ value (here $x=0$ is chosen). It appears that with increasing

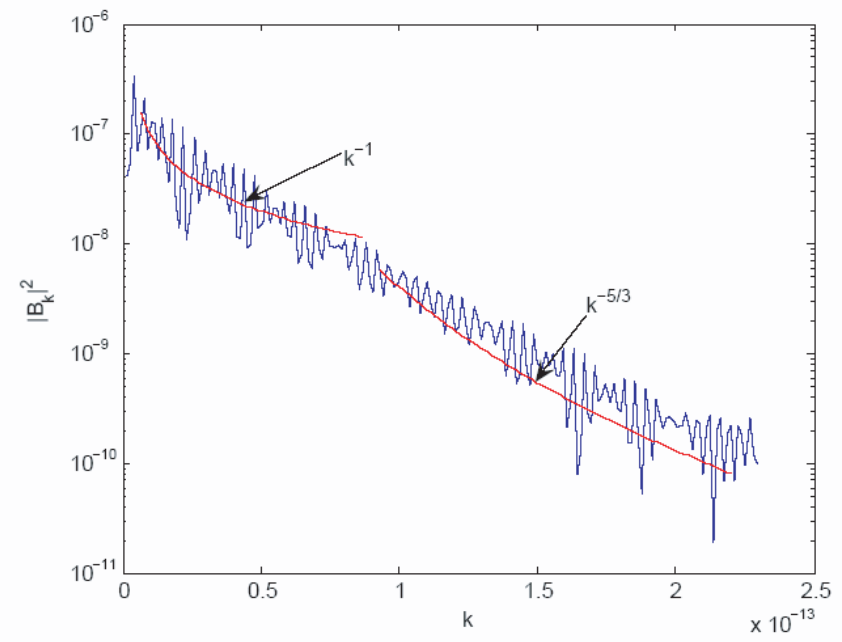

Fig. 5. $\left|B_{k}\right|^{2}$ against $k$ at $x=0$ in the presence of second KAW.

values of the $k$, the scaling of the spectra changes from the $k^{-1}$ towards Kolmogorov scaling $k^{-5 / 3}$.

Acknowledgements. This work is partially supported by DST (India). M. Malik is very grateful to CSIR for providing financial assistance for the present work.

\section{References}

Akhmanov, S. A., Sukhorukov, A. P., \& Khokhlov, R. V. 1968, Sov. Phys. Uspekhi, 10, 609

Bavassano, B., Dobrowolny, M., Mariani, P., \& Ness, N. F. 1982, J. Geophys. Res., 87,3617

Belcher, J. W., \& Davis, L. 1971, J. Geophys. Res., 76, 3534

Bellan, P. M., \& Stasiewicz, K. L.1998, Phys. Rev. Lett., 80, 3523.0

Champeaux, S., Passot, T., \& Sulem, P. L. 1997, J. Plasma Phys., 58, 665

Champeaux, S., Passot, T., \& Sulem, P. L. 1998, Phys. Plasmas, 5, 100

Coleman, P. J., Jr. 1966, Phys. Rev. Lett., 17, 207

Cranmer, S. R., Field, G. B., \& Kohl, J. L. 1999, ApJ, 518, 937

Czechowski, A., Ratkiewicz, R., McKenzie, J. F., \& Axford, W. I. 1998, A\&A, 335,303

Daily, W. D. 1973, J. Geophys. Res., 78, 2043

Dendy, R. O. 1995 (Cambridge University Press)

Denskat, K., \& Burlaga, L. F. 1977, J. Geophys. Res., 82, 2693

Dusenbery, P. B., \& Hollweg, 1981, J. Geophys. Res., 86, 153

Fairfield, D. H. 1969, J. Geophys. Res., 74, 3541

Gekelman, W. 1999, J. Geophys. Res., 104, 14417

Hasegawa, A., \& Chen, L. 1975, Phys. Rev. Lett., 35, 370

Hollweg, J. V., \& Isenberg, P. A. 2002, J. Geophys. Res., 107 (A7)

Hollweg, J. V., \& Turner, J. M. 1978, J. Geophys. Res., 83, 97

Hollweg, J. V., Bird, M. K., Volland, H., et al. 1982, J. Geophys. Res., 87, 1

Hoppe, M. M., \& Russell, C. T. 1983, J. Geophys. Res., 88, 2021

Hu, Y. Q., Esser, R., \& Habbal, S. R. 1997, J. Geophys. Res., 102, 14661

Isenberg, P. A. 1984, J. Geophys. Res., 89, 6613

Jokipii, J. R. 1973, ARA\&A, ed. L. Goldberg, D. Layzer, \& J. G. Philip, 11, 1

Leer, E., Holzer, T. E., \& Shoub, E. C. 1992, J. Geophys. Res., 97, 8183

Louarn, P., Wahlund, J. E., Chust, T., et al. 1994, Geophys. Res. Lett., 21, 1847

Lysak, R. L., \& Lotko, W. 1996, J. Geophys. Res., 101, 5085

Marsch, E., Goertz, C. K., \& Richter, K. 1982c, J. Geophys. Res., 87, 5030

Parker, G. D. 1980a, J. Geophys. Res., 85, 4275

Parker, G. D. 1980b, J. Geophys. Res., 85, 4283

Parker, E. N. 1991, ApJ, 376, 355

Shukla, A., \& Sharma, R. P. 2000, J. Geophys. Res., 107, 1338

Shukla, A., Sharma R. P., \& Malik, M. 2004, Phys. Plasmas, 11, 2068

Shukla, P. K., \& Stenflo, L. 1999, Phys. Plasmas, 6, 4120

Shukla, P. K., \& Stenflo, L. 2000, Phys. Plasmas, 7, 2738

Stasiewicz, K., Bellan, P., Chaston, C., et al. 2000, Space Sci. Rev., 92, 423

Tsiklauri, D., Sakai, J. -I., \& Saito, S. 2005, A\&A, 435, 1105

Unti, T. W., \& Neugebauer, N. 1968, Phys. Fluids, 11, 563

Wahlund, J., Louarn, P., Chust, T., et al. 1994, Geophys. Res. Lett., 21, 1831

Wu, D. J. 2003, Commun. Theor. Phys., 39, 457 